\title{
Improving medication adherence of patients with chronic heart failure: challenges and solutions
}

\author{
This article was published in the following Dove Press journal: \\ Research Reports in Clinical Cardiology \\ 15 July 2015 \\ Number of times this article has been viewed
}

\section{Deval Shah' \\ Kim Simms ${ }^{2}$ \\ Debra J Barksdale ${ }^{3}$ \\ Jia-Rong $\mathrm{Wu}^{3}$}

'Internal Medicine, Wake Forest Baptist Hospital, Winston-Salem, ${ }^{2}$ Duke University Hospital, Durham, ${ }^{3}$ School of Nursing, University of North Carolina, Chapel Hill, NC, USA
Correspondence: Deval Shah Internal Medicine, Wake Forest Baptist Hospital, I Medical Center Boulevard, Winston Salem, NC 27I03, USA

Tel +l 7048774679

Email dshah1217@gmail.com

\begin{abstract}
Heart failure is a chronic debilitating illness that affects 5.7 million Americans. The financial burden of heart failure in the US toppled \$31 billion in 2012, which is one of the highest among all chronic medical conditions. Medication adherence is a major component of heart failure self-care behaviors. Therefore, medication non-adherence is associated with more emergency department visits, frequent rehospitalizations, and higher medical cost. Medication adherence rates have varied from $10 \%$ to $98 \%$ depending on the definition and measurement used to assess and analyze adherence. Many factors contribute to medication non-adherence such as lack of support, finances, absent of symptoms, cognitive decline, adverse reactions, depression, poor attention span, poor knowledge about medication, multiple medications, difficulty swallowing large pills, and inconveniences of urinary frequency with diuretics Researchers have explored various strategies such as the use of pharmacists, nurses, telemedicine, and interdisciplinary teams to provide interventions to improve medication adherence in heart failure. Health care providers should continue to provide education, constantly reinforce the importance of taking medication as prescribed, and when feasible, utilize one of the successful evidence-based strategies to increase adherence.
\end{abstract}

Keywords: pharmacy, tele-health, interdisciplinary, registered nurse, interventions

\section{Background}

In the US, 5.7 million people are living with heart failure. ${ }^{1}$ About half of the patients with heart failure will die in the first 5 years after being diagnosed. ${ }^{1}$ In heart failure, the heart does not supply adequate blood flow to meet the body's demands. ${ }^{2}$ Common signs and symptoms of heart failure include exertional dyspnea, orthopnea, peripheral edema, reduced exercise tolerance, paroxysmal nocturnal dyspnea, and weight gain. ${ }^{2}$ Managing heart failure is complex, and this chronic condition has a 30-day hospital readmission rate of $27 \%$, which is one of the highest among all chronic medical conditions. ${ }^{3}$ Rates have also been reported to be as high as $25 \%$ of previously hospitalized patients who are readmitted in 6-9 months for heart failure exacerbations. ${ }^{4}$

The financial burden of heart failure in the US toppled $\$ 31$ billion in 2012, which is one of the highest among chronic medical conditions. ${ }^{5}$ These costs include not only medications but also health care services and lost productivity. The average cost of a heart-failure related hospitalization in 2005 was $\$ 23,077$ and was higher when heart failure was coupled with other chronic conditions that exacerbate heart failure symptoms such as respiratory illnesses, ischemic heart disease, and other heart diseases. ${ }^{6}$ Hospital readmissions are not only an important indicator of health outcomes of patients with heart failure but also lead to increased cost of care. 
A patient's ability to perform self-care is crucial to the overall management of heart failure and is needed at all stages of the disease. ${ }^{7}$ Self-care behaviors help decrease all-cause hospital admission and readmission for heart failure exacerbations. ${ }^{8}$ Medication adherence is the most important self-care behavior to prevent acute exacerbations of heart failure. ${ }^{2,9}$ Therefore, the focus of this review is on challenges and solutions to promote the self-care behavior of medication adherence. We discuss the importance of medication adherence and also barriers to medication adherence.

\section{Challenges of medication adherence}

The treatment plan for patients with heart failure is multifaceted and complex. The primary treatment for heart failure is pharmacological therapy. ${ }^{2}$ Drug therapies for heart failure are important in controlling symptoms, slowing the progression of cardiac remodeling, decreasing readmission rate, and improving survival rate. ${ }^{10}$ Patients who follow their prescribed regimen can decrease their risk of hospitalization and death. For example, in a study by Wu et al, patients who were adherent to medications had no adverse events for more consecutive days than patients who were non-adherent. ${ }^{11}$ In another study, patients who did not adhere to their prescribed medication regimen were two times as likely to be admitted to hospital and three times as likely to experience death compared to patients who were adherent to their regimens. ${ }^{12}$ Moreover, when patients are non-adherent to their drug therapy, symptoms are less likely to be controlled. ${ }^{10}$ These studies indicate the importance of following prescribed medication regimens to decrease adverse events in heart failure. ${ }^{10-12}$ In the following section, we discuss challenges and factors that affect adherence to medication.

Poor adherence to medications as prescribed is the first challenge. ${ }^{13}$ Rate of adherence reported in heart failure studies has varied from $10 \%$ to $98 \%$ depending on the measuring instruments used, and most authors have noted an average of $40 \%-60 \%$ of medication adherence. ${ }^{14}$ In addition to low medication adherence rates, medication adherence has been difficult to study in patients with heart failure because researchers have varied measures to assess medication adherence. It has been measured using subjective reports (self-reports) and objective measurements (pill counts, pharmacy refill records, and electronic medication-monitoring systems). ${ }^{15}$ Medication adherence rates assessed from selfreports were reported to be anywhere from $78 \%$ to $100 \%$ in four studies. ${ }^{16-19}$ Investigators used a variety of questionnaires to measure adherence (eg, Revised Heart Failure Compliance Questionnaire). ${ }^{16-19}$ Adherence was measured by arbitrary cutoff points $(80 \%) .{ }^{20}$ Patients' self-report of their adherence can introduce bias due to inaccurate recall or reporting an overly optimistic estimation of adherence to the health care providers. ${ }^{20}$

Pill counts are one of the objective ways to measure medication adherence. For pill counts, the pills being studied are counted, and this number is compared with the prescription refill date. Measured using pill count, the adherence rates ranged from $85 \%$ to $99 \%$ in four studies. ${ }^{21-24} \mathrm{~A}$ limitation with pill count is that it may not be representative of the long-term adherence because patients may exhibit white-coat adherence which is improved medication-taking behaviors a few days before and after seeing a health care provider. ${ }^{25}$ Another limitation with pill count is that it is not known if the patient actually took the pill. ${ }^{25}$ Pharmacy refill is another objective way to measure adherence. Refill data are obtained from a pharmacy database. When pharmacy refill was used as a determinant, the medication adherence ranged from $77 \%$ to $81 \%$ in three studies. ${ }^{26-28}$ There are two ways to measure pharmacy refill history to assess medication adherence. The first way is called the medication possession ratio. ${ }^{15}$ This ratio compares the number of days a medication is available with the total length of time in a study (number of days medication available/total length of days in study). The second way is called the cumulative mean gap ratio. ${ }^{15}$ In this ratio, the numerator is total number of days the medication is unavailable, and the denominator is total length of time patients are in the study. Each investigator used different methods to assess medication adherence. Also, all of these studies had different cutoff points (80\%-88\%) for defining adherence; so, this made it difficult to compare results between studies.

A medication event-monitoring system (MEMS) device is an electronic monitoring system that can be utilized to assess adherence objectively. There is a chip inside the bottle cap which records every time the cap is opened. This system can provide information about percentage of prescribed doses taken, dose taken at correct time, and correct number of doses taken. The adherence rates measured using this method ranged from $65 \%$ to $94 \%$ in five studies. ${ }^{29-33}$ MEMS devices cannot assess whether a patient took the pill once the bottle was opened which limits its utilization. ${ }^{15}$ Other limitations of MEMS device are that it is too expensive, only one prescription can be used in one MEMS bottle, and many patients are using pill box.

In summary, in these studies, medication adherence rates were dependent on the type of method used and the definition of adherence used by the researchers. Adherence was 
measured using arbitrary cutoff point, so further research is needed to determine the level of adherence.

\section{Factors of medication adherence}

Failure to adhere to a prescribed medication regimen is affected by multiple and complex factors. There are many ways to group these factors. For example, we can group these factors as intentional and unintentional. Intentional non-adherence includes factors that patients take an active role with their own beliefs and reasons and decide to follow or disregard professional advice, ${ }^{34}$ and is studied with social cognition model (theory of planned behavior and cognitive therapy). ${ }^{34}$ The determinants of person's behavior comprise attitudes (beliefs about outcomes of adherences to medication), subjective norm (patient's significant others and physician approve or disapprove of medication adherence), and perceived behavioral control (availability of resources for adherence to medication). ${ }^{34}$ Unintentional non-adherence is less likely to be associated with individuals' intention to be non-adherent. ${ }^{34}$ Unintentional non-adherence can be related to patient factors (age, sex, ethnicity, socioeconomic status, psychological distress), treatment factors (increase in frequency of dosing, increase in number of medications), and patient-provider factors (quality of the relationship, behavior and attitudes of health care professional, and time spent with patients). ${ }^{34}$ Assessing intentional and unintentional dimensions of medication-taking behaviors may help the clinicians to better intervene medication non-adherence. However, there is still no good assessment tool to clearly identify which factors are intentional and unintentional, and the concept of intentional and unintentional factors of medication nonadherence is still underdeveloped.

Another way is to group these factors into a five-dimension adherence model from the World Health Organization report on adherence to long-term therapies to guide this paper: health system-related, socioeconomic, condition-related, therapy-related, and patient-related. ${ }^{35}$ Health system-related factors could include lack of training of health care providers in chronic illness, shortened time for patient office visits, and lack of reimbursement from insurance providers. ${ }^{35}$ Further, positive patient-provider relationships are associated with greater adherence to the medication regimens. ${ }^{36}$

Age, sex, race, and lack of access to transportation contribute to socioeconomic factors that affect medication adherence. ${ }^{35}$ Lack of support and finances are often cited as factors that influence adherence. ${ }^{26}$ As drug co-payments increase, medication adherence rates decrease leading to an increased risk of hospitalization. ${ }^{37}$ With every ten-dollar increase in co-payment for an angiotensin-converting enzyme inhibitor, there was a $2.6 \%$ decrease in adherence rate with a $6.1 \%$ increase in the risk of hospitalization. ${ }^{37}$

Condition-related factors such as severity of illness and depression could influence patients' ability to follow their prescribed treatment regimen. ${ }^{35}$ Patients who have depression have a greater likelihood of non-adherence. ${ }^{38}$ In a study by Cholowski and Cantwell, patients with depressive symptoms were $15 \%$ less likely to adhere to a prescribed regimen. ${ }^{38}$ Also, additional medical comorbidities can affect adherence rates. In two studies, it was noted that with an increase in comorbidities, medication adherence rates decrease. ${ }^{39,40}$

The therapy-related factors that influence adherence include difficulty swallowing large pills, inconvenience of urinary frequency while on diuretics, and multiple medications needed throughout the day. ${ }^{36}$ As the number of pills taken per day is increased, medication adherence increases. ${ }^{41,42}$ Also, patients are more likely to skip a dose when they experience side effects. ${ }^{41,42}$

Patient-related factors that affect medication adherence include forgetting, feeling that treatment has "succeeded" as evidenced by decreased or absent symptoms, excessive daytime sleepiness, poor attention span, and cognitive decline. ${ }^{35,39,43}$ Also, patients who do not have adequate knowledge about their medication regimen were more likely to be non-adherent. ${ }^{10}$

The challenge of medication adherence is multifactorial, influenced by the factors listed above. These factors cause difficulty for the patients to adhere to their heart failure medication regimen. It would be best to develop interventions to address these factors to improve medication adherence.

\section{Possible solutions}

To overcome the challenges of low medication adherence and other factors that impede adherence, many researchers have developed and tested interventions for improving medication adherence. In this integrative review of the research to identify interventions, CINAHL and PubMed were searched. Search terms included chronic heart failure, self-care, self-management, and medication adherence. Inclusion criteria were as follows: 1) randomized controlled trial or interventional study, 2) published between the years 1995 and 2014, 3) published in English language, 4) primary diagnosis of heart failure, and 5) with a primary outcome to increase medication adherence. Articles were excluded if they focused on a diagnosis other than heart failure or a primary outcome that did not include medication 
adherence. There were eleven published papers reporting the findings of interventional studies on improving medication adherence and subsequent hospitalizations of patients with heart failure. The results from these studies provide insight on the current status of medication adherence. For purposes of this paper, these studies were grouped into four categories: 1) pharmacist-led education, ${ }^{16,22,29,33}$ 2) registered nurse- or case mangers-led education, ${ }^{21,23,32} 3$ ) usage of tele-health or web-based modules, ${ }^{19,30,31}$ and 4) interdisciplinary team-led education. $^{24}$

\section{Pharmacist-led educational interventions}

Pharmacists have many roles in clinical settings. Firstly, pharmacists can provide education about the importance of medications, side-effects, and the relationship with the medical condition. ${ }^{30}$ Secondly, pharmacists can contact the physician if there are any interactions among various medications in the patient's profile. ${ }^{30}$ Thirdly, pharmacists can contact patients who have not refilled their prescriptions. ${ }^{30}$ Since health care providers may not be able to spend extensive time with the patients, pharmacists can be essential resources for counseling patients about their medication. ${ }^{30}$ Therefore, there are many ways a pharmacist can help improve medication adherence. For example, pharmacists can help address patient-related factors of non-adherence if patients are consistently forgetting to take their medication by giving them a pill-reminder box. They can address socioeconomic factors by being a liaison with the patient's primary care provider to assist in recommending a generic medication within the same class of medications. Moreover, pharmacists can address treatment-related factors by asking the patients if it is difficult to swallow pills or take too many pills, or if there are any side effects. Pharmacists can address the issue by having a discussion with the patient's primary care provider to come up with a solution. There were four randomized controlled trials analyzing the efficacy of a pharmacist delivering education about medications to patients with heart failure. ${ }^{16,22,29,33}$

In a prospective randomized controlled study by Cabezas et al, pharmacists delivered a year-long educational intervention to 134 participants after discharge to improve adherence rates. $^{22}$ In this study, 70 participants were assigned to the intervention group and 64 to the control group. The intervention group received education from the pharmacist and telephone follow-up every month for the first 6 months and every 2 months for the remainder of the study. The control group received regular care with a telephone follow-up. Medication adherence rates were measured by pill count. The patients were classified as reliable (taking 95\%-100\% of prescribed doses), partially reliable (85\%-95\%), and not reliable $(<85 \%)$. The intervention group had a medication adherence rate of $85 \%$ at 12 months, while the control group had an adherence rate of $73.9 \%$. Also, the intervention group had only 410 days of hospital stay, while the control group had a total of 611 days of hospital stay. The differences in medication adherence and hospital days were not significant for this study due to positive effect on participating in a research study. Participants in the intervention group had a lower mortality rate than those in the control group (29.7\% vs $12.9 \%, P=0.017) .^{22}$

In another randomized controlled trial, pharmacists delivered an educational intervention on loop diuretics usage to patients who attended a heart failure clinic in the Netherlands. ${ }^{29}$ The medication adherence rate was measured using the MEMS device. There were 74 patients in the intervention group and 78 in the control group. ${ }^{29}$ No participants in the intervention group had less than $80 \%$ adherence, but six participants in the control group were less than $80 \%$ adherent to their loop diuretics regimen. The relative risk of non-adherence to medication ( $<80 \%$ adherence) between both groups was 0.5 , indicating that the control group was more likely to not adhere to prescribed medication than the intervention group $(P<0.05) .{ }^{29}$ In another study, a pharmacist provided an educational booklet and self-monitoring program for heart failure (signs and symptoms of heart failure exacerbations and adherence to medication) to the intervention group $(n=109) .{ }^{16}$ The control group $(n=112)$ received traditional education (education in the clinic) from their physician or nurse practitioner. Participants' adherence rates were self-reported. Non-adherence to medication, in this study, was also defined as taking an extra dose of the medication without medical advice. In the intervention group, 85 out of 109 participants reported adherence, while only 35 out of 112 patients in the control group reported that they were adherent to their medications $(P<0.05)$. The number of hospitalizations in the intervention group was significantly lower than in the control group (22 in intervention group vs 36 in control group). ${ }^{16}$

In a study by Murray et al, a pharmacist provided verbal and written education about medication to the intervention group $(n=122) .{ }^{33}$ The control group $(n=192)$ did not receive any education from the pharmacist. Participants were recruited from general medicine and cardiology offices. ${ }^{33}$ Medication adherence was measured with the MEMS device. There was higher adherence in the intervention group compared to the control group at the end of intervention period (78.8\% vs $67.9 \%$ ). There were $19.8 \%$ 
fewer hospitalizations in the intervention group than control group. ${ }^{33}$ Difference between groups in medication adherence and hospitalization rates was statistically significant per author report, but no $P$-values were stated.

The intervention groups in the four studies received education from the pharmacists, while the control group received education as usual care. The adherence rate was measured by pill count ${ }^{22}$ or MEMS device ${ }^{29,33}$ or self-reported. ${ }^{16}$ When pharmacists provided education, the participants in interventions groups were more likely to adhere to their medications regimen than participants in the control groups. ${ }^{16,22,29,33}$ In two studies, participants in the intervention groups had decreased incidence of hospitalizations than the control groups (19.8\%). ${ }^{16,33}$ Therefore, pharmacist-led educational interventions can be effective at promoting medication adherence and a good solution to improving medication adherence of patients with heart failure.

\section{Registered nurse- and case management-led interventions}

Nurses can be an important health care provider assisting to increase medication adherence. For example, nurses often have a personal connection with the patients before they receive any medication regimen. ${ }^{44}$ This personal connection helps patients to better accept nurses' education about the risks and benefits of taking their medication as prescribed. ${ }^{44}$ Nurses often utilize the teach-back method to affirm patient's understanding of the instructions, ${ }^{44}$ which addresses an important patient-related factor for medication adherence by increasing their knowledge. Three studies explored the effect of either a registered nurse- or a case manager-led intervention on self-care behaviors for heart failure. ${ }^{21,23,32}$

In a study by Barnason et al, a registered nurse led an education intervention for heart failure patients who were hospitalized and provided telephone follow-up and education 2-3 weeks post-discharge. ${ }^{21}$ The participants in the control group received the same education as the intervention group from the nurse at the hospital but did not receive any postdischarge follow-up education. ${ }^{21}$ There were 40 participants in the study - 20 in the intervention group and 18 in the control group with two participants who did not complete the study. ${ }^{21}$ Medication adherence was measured with the Brief Medication Questionnaire. The nurses asked a series of questions related to taking prescribed doses and how many doses were missed. Non-adherence was determined when a patient reported missing $>20 \%$ of dosages. Those in the intervention group had medication adherence of $99.1 \%$ at 1 month and $99.3 \%$ at 3 months. ${ }^{21}$ Comparatively, the control group had an adherence rate of $83.2 \%$ at 1 month and $86.4 \%$ at 3 months. ${ }^{21}$ The intervention group had significantly higher adherence rates than the control group $(P<0.001) .{ }^{21}$ In another study, a case manager, who was a registered nurse, delivered an education program about heart failure to patients who were admitted to the hospital. ${ }^{23}$ The intervention group $(n=122)$ received a post-discharge phone call weekly for the first 4 weeks and then bi-weekly phone calls until the 12 th week. This was along with follow-up by their physician in the outpatient setting. The control group $(n=112)$ received education at the hospital about heart failure management and were followed up by their physician in the outpatient setting. ${ }^{23}$ Patients in the intervention group experienced fewer days of hospitalization than in the control group (6.9 days vs 9.5 days, $P=0.15){ }^{23}$ There was no statistically significant difference in number of days between groups because intervention group had higher outpatient costs and were less likely to be seen by a cardiologist. Even though both groups adhered to their medication equally, the intervention group did show improvement in adherence to other self-care behaviors (weighing daily, checking for edema, low-salt diet, and fluid restriction) $(P<0.01){ }^{23}$

Lastly, Wu et al conducted a study using the theory of planned behavior to guide education about symptoms of heart failure and the importance of adhering to medications. ${ }^{32}$ There were 82 patients recruited to participate in the study from an outpatient cardiology clinic and inpatient hospital in one southern state. ${ }^{32}$ All participants were given an MEMS device which was used to measure medication adherence. ${ }^{32}$ There were two intervention groups (PLUS and LITE groups) and one control group. Both the PLUS (n=27) and LITE groups $(n=27)$ received education about heart failure. In addition, the PLUS group received feedback about their medication adherence at various intervals during the study. The control group $(n=28)$ did not receive any education about heart failure. Patients were called every month for 9 months to obtain hospitalization data. At baseline, there was no significant difference in adherence rate among the three groups (PLUS - 70\%, LITE - 59\%, control - 64\%, $P=0.694) .{ }^{32}$ The reason for no difference in adherence among three groups at baseline was not reported. At 9 months, the patients in the PLUS group had better medication adherence compared to the other two groups (PLUS - 74\%, LITE - 65\%, control $-36 \%, P=0.015) .{ }^{32}$ At the end of the intervention, there were significant differences between both intervention and control groups in the hospitalization rate $(P<0.01) .{ }^{32}$ All the studies presented that they utilized education to improve adherence. ${ }^{21,23,32}$ One of the study even used theory 
of planned behavior which helped to improve adherence and address intentional factors of non-adherence. ${ }^{32}$ The adherence rate was measured by adherence questionnaire, ${ }^{21}$ telephone follow-up, ${ }^{23}$ or MEMS device. ${ }^{32}$ From the three studies presented, two studies had the greatest effect in improving medication adherence (Barnason, $P<0.01 ; \mathrm{Wu}, P=0.015) .{ }^{21,32}$ In another study, participants improved in other self-care behaviors (weighing daily, checking for edema, and low-salt diet) (Laramee, $P<0.01) .{ }^{23}$ The education provided by the nurses can be effective at improving medication adherence and increasing adherence to other self-care behaviors.

\section{Tele-health and web-based interventions}

Telemedicine is defined as when medical information is exchanged via electronic communications. ${ }^{45}$ There are a variety of services and applications that are offered where provider and patient can exchange information over the Internet. Telemedicine can be an effective way to deliver education because patients can be anywhere as long as they have access to a telephone and the Internet. ${ }^{45}$ The education delivered via telemedicine can be provided by members of multidisciplinary health teams such as pharmacist, nurses, dieticians, and physicians. ${ }^{45}$ The education received can help address patient-, health system-, and socioeconomic-related factors impacting adherence. It would address patient-related factors because patients would have increased knowledge about their condition, and with increased knowledge, they are more likely to adhere to their treatment regimen. It can help address health system-related factors because of close interactions with providers that create positive patient-provider relationship. In addition, telemedicine may diminish transportation and access issues and help address socioeconomic-related factors for medication non-adherence. Three studies examined usage of tele-health or web-based interventions to increase medication adherence. ${ }^{19,30,31}$

In the study by Fulmer et al, there were three groups (one control group and two intervention groups). ${ }^{31}$ Both intervention groups received daily calls reminding them to take their medications. One intervention group received daily telephone calls, and the other intervention group received video-telephone call. The control group received the usual care. The participants were recruited from one home health agency and one ambulatory clinic in New York. The adherence rate was measured with MEMS caps. The baseline adherence rates for the three groups were as follows: control group $-81 \%$, telephone group $-76 \%$, and video-telephone group $-82 \%$. After 6 weeks of the inter- vention, the adherence rate in the control group dropped dramatically to $57 \%$. In the telephone intervention group, the adherence rate was $74 \%$, and the adherence rate in the video-telephone group was $84 \%$. The difference between group adherence rates was statistically significant.

In the study by Artinian et al, 18 patients from a Veteran's Affairs heart failure clinic were enrolled in the intervention $(n=9)$ and control groups $(n=9) .{ }^{30}$ All participants received a weight scale and blood pressure (BP) machine. The patients in the intervention group received web-based modules and a medication compliance device, Med-eMonitor, to measure medication adherence. ${ }^{30}$ The participants in the intervention group had a $94 \%$ medication compliance rate, $85 \%$ weight-monitoring compliance rate, and $81 \%$ BP-monitoring compliance rate. ${ }^{32}$ Medication adherence was measured by pill counts for the control group. ${ }^{30}$ Weight-monitoring and BP-monitoring compliance were measured by patients' $\log .{ }^{30}$ There were no data available for the medication adherence in the control group because patients did not follow directions correctly according to the researchers. ${ }^{30}$ However, researchers did not specify which directions were not followed. ${ }^{30}$ In the control group, the adherence rate for weight monitoring was $79 \%$ and BP monitoring was 51\%. ${ }^{30}$ In the study by Wakefield et al, participants from a Veterans Affairs Medical Center who were recently discharged from the hospital with congestive heart failure were recruited for the study. ${ }^{19}$ The participants were randomized to one of three groups: usual care $(n=49)$, telephone group $(n=47)$, and videophone $(n=52)$. The investigators evaluated the efficacy of treatment adherence post-hospital discharge. The participants in the intervention groups were contacted weekly for 90 days after discharge to remind them to take their medication. ${ }^{19}$ However, there was no difference between intervention and control groups in medication adherence. ${ }^{19}$ Although there was no difference in medication adherence between the intervention and control groups, participants in the intervention groups were able to correctly state the purpose and side effect of the medication about $90 \%$ of the time at the end of the intervention. ${ }^{19}$ There were no data reported for correctly stating the purpose and side effects of the medications for the control group. ${ }^{19}$

From the studies presented for tele-health, intervention was received via phone call ${ }^{19,31}$ or web-based modules. ${ }^{30}$ The adherence rate was self-report ${ }^{19}$ or measured by compliance device (Med-eMonitor, MEMS device). ${ }^{30,31}$ From the three studies presented, only one of the studies showed improvement in medication adherence (videophone- $84 \%$, telephone- $74 \%$, control group $-57 \%) .{ }^{31}$ Therefore, more studies are needed 
to further evaluate the effectiveness of telemedicine in improving medication adherence in heart failure patients.

\section{Interdisciplinary team approach intervention}

Utilization of an interdisciplinary team can be effective in delivering education about medication adherence. The interdisciplinary team could consist of a nurse, pharmacist, registered dietician, social worker, and a physician. The interdisciplinary team would assist the patient in understanding the relationship between medication, diet, and symptoms. ${ }^{24}$ Dietician would provide in-depth education on dietary recommendations. The social worker would assist in appropriate post-discharge care and can help address socioeconomic-related factors by verifying that patient has appropriate low-cost medication resources. ${ }^{24}$ The physician and pharmacist roles are to ensure that the patient is on the correct medicine and there are no interactions with other medications and would help address therapy- and condition-related factors. The nurse role would be to provide education about heart failure along with medication (in collaboration with the pharmacist) and to reinforce dietary recommendations in collaboration with the dietitian. The education provided by interdisciplinary team would help address patient-related factors by increasing patient's knowledge. This interdisciplinary approach reinforces the treatment regimen and provides resources to prevent readmission to hospital. ${ }^{24}$

One study addressed utilization of an interdisciplinary team approach to educate patients about heart failure management and its effects on medication adherence. The intervention group $(\mathrm{n}=80)$ received education from the study nurses, a dietician, social worker, and a geriatric cardiologist. ${ }^{24}$ During the hospital stay, the study nurse taught the importance of adherence to medication and diet every day. The dietician provided additional dietary instructions, and the social worker made arrangements for post-discharge plan. Shortly before discharge, the geriatric cardiologist made specific recommendations to simplify and consolidate the number of medications and dosing frequency. Ultimately, the primary physician would decide whether or not to follow the recommendations. The control group $(n=76)$ received conventional education under the direction of a primary physician. ${ }^{24}$ The participants in the control group received education about diet and discharge medication instructions. After discharge, every participant in the study received a call from a study nurse regularly, and a pharmacist visited every participant's home. The adherence rate was assessed by pill count. ${ }^{24}$ Patients in the intervention group had a significantly higher adherence to their medication regimen than the control group ( $87 \%$ vs $80 \%, P=0.004) .{ }^{24}$ The participants in the control group had more hospitalizations than those in the intervention group (31 vs 22) although not statistically significant. ${ }^{24}$ The reason for statistically nonsignificant readmission is effect sizes.

We located one study on the interdisciplinary approach that increased medication adherence rates in heart failure. ${ }^{24}$ In this study, the number of hospitalizations in the intervention group was fewer than control group. The interdisciplinary approach can be excellent in providing education to patients about heart failure and their medications. However, more studies are needed to assess the effectiveness of this approach in improving medication adherence in patients with heart failure.

\section{Conclusion}

Despite advances in heart failure treatment over the past several decades, the number of individuals affected by poor medication adherence remains high. Medication adherence is an important modifiable factor in reducing poor health outcomes and costs from acute exacerbations in patients with heart failure. ${ }^{8}$ Challenges and barriers to medication adherence are complex and multidimensional. However, the identification and removal of these barriers have been shown to be effective in increasing medication adherence and reducing length of stay. Most of the intervention studies we found during the search had an educational component. Education is a prerequisite, not sufficient. Due to lack of understanding of the importance of medication adherence, patients need educational counseling to help them increase their knowledge. Pharmacists and nurses have their unique roles, contributions, and connections to provide education to help patients understand the importance and strategies to overcome their barriers. In addition, they can be the channel/bridge to communication between patients and their physicians to adjust patients' prescriptions if any problems arise. From our review, interdisciplinary team approach would be the best way to improve medication adherence, since the patient would receive education and resources from every multiple disciplines. This would help patient make connections with symptoms, medications, and the diet. However, when considering cost involved with interdisciplinary approach, pharmacists or nurses can provide an effective education on improving medication adherence and decreasing hospitalization rates. Lastly, tele-health is an excellent option to consider, but it is not an option at this moment to everyone 
due to lack of access to Internet. Health care providers should continue to provide education to patients about the importance of taking medications as prescribed, and when feasible, utilize one of the successful strategies presented to increase medication adherence.

\section{Acknowledgments}

This paper was supported by funding from the National Institute of Nursing Research of the National Institutes of Health under Award Number K23NR014489 (Jia-Rong Wu, principal investigator). The funding body did not play a role in the writing of the manuscript or the submission of the manuscript for publication. The content is solely the responsibility of the authors and does not necessarily represent the official views of the National Institute of Nursing Research or the National Institutes of Health.

\section{Disclosure}

The authors of this work state that there are no conflicts of interest associated with this paper.

\section{References}

1. Mozaffarian D, Benjamin E, Go AS, et al. Executive summary: heart disease and stroke statistics - 2015 update: a report from the American heart association. Circulation. 2015;131:e29-e322.

2. Kemp C, Conte J. The pathophysiology of heart failure. Cardiovasc Pathol. 2012;21(5):365-371.

3. Jencks SF, Williams MV, Coleman EA. Rehospitalizations among patients in the Medicare fee-for-service program. J Vasc Surg. 2009; 360:1418-1428.

4. Alehagen U, Rahmqvist M, Paulsson T, Levin L. Quality-adjusted life year weights among elderly patients with heart failure. Eur J Heart Fail. 2008;10(10):1033-1039.

5. Heidenreich P, Maddox T, Nichol G. Forecasting the impact of heart failure in the United States: a policy statement from the American Heart Association. Circ Heart Fail. 2013;6(3):606-619.

6. Wang G, Zhang Z, Ayala C, Dunet D, Fang J. Costs of heart failurerelated hospitalizations in patients aged 18 to 64 years. Am J Manag Care. 2010;16(10):769-776.

7. Riegel B, Lee CS, Dickson VV. Self care in patients with chronic heart failure. Nat Rev Cardiol. 2011;8(11):644-654.

8. Jovicic A, Holroyd-Leduc JM, Straus SE. Effects of self-management intervention on health outcomes of patients with heart failure: a systematic review of randomized controlled trials. BMC Cardiovasc Disord. 2006;6:43.

9. O'Connor CM, Stough WG, Gallup DS, Hasselblad V, Gheorghiade M. Demographics, clinical characteristics, and outcomes of patients hospitalized for decompensated heart failure: observations from the IMPACT-HF registry. J Card Fail. 2005;11(3):200-205.

10. Hope C, Wu J, Tu W, Young J, Murray M. Association of medication adherence, knowledge, and skills with emergency department visits by adults 50 years or older with congestive heart failure. Am J Health Syst Pharm. 2004;61(19):2043-2049.

11. Wu J, Moser DK, Chung ML, Lennie TA. Objectively measured, but not self-reported, medication adherence independently predicts event-free survival in patients with heart failure. J Card Fail. 2008;14(3):203-210.

12. Fitzgerald AA, Powers JD, Ho PM, et al. Impact of medication nonadherence on hospitalizations and mortality in heart failure. J Card Fail. 2011;17(8):664-669.
13. Corotto PS, McCarey MM, Adams S, Khazanie P, Whellan DJ. Heart failure patient adherence: epidemiology, cause, and treatment. Heart Fail Clin. 2013;9(1):49-58.

14. Oosterom-Calo R, van Ballegooijen AJ, Terwee CB, et al. Determinants of adherence to heart failure medication: a systematic literature review. Heart Fail Rev. 2013;18(4):409-427.

15. Wu J, Moser DK, Lennie TA, Burkhart PV. Medication adherence in patients who have heart failure: a review of the literature. Nurs Clin North Am. 2008;43(1):133-153.

16. Sadik A, Yousif M, McElnay J. Pharmaceutical care of patients with heart failure. Br J Clin Pharmacol. 2005;60(2):183-193.

17. Nieuwenhuis MM, Jaarsma T, van Veldhuisen DJ, van der Wal MH. Self-reported versus 'true' adherence in heart failure patients: a study using the medication event monitoring system. Neth Heart J. 2012; 20(7):313-319.

18. González B, Lupón J, Parajón T, et al. Nurse evaluation of patients in a new multidisciplinary heart failure unit in Spain. Eur J Cardiovasc Nurs. 2004;3(1):61-69.

19. Wakefield B, Holman J, Ray A, et al. Outcomes of a home telehealth intervention for patients with heart failure. J Telmed Telcare. 2009; 15(1):46-50.

20. Ho PM, Bryson CL, Rumsfeld JS. Medication adherence: its importance in cardiovascular outcomes. Circulation. 2009;119(23):3028-3035.

21. Barnason S, Zimmerman L, Hertzog M, Schulz P. Pilot testing of a medication self-management transition intervention for heart failure patients. West J Nurs Res. 2010;32(7):849-870.

22. Cabezas C, Salvador C, Quadrada D. Randomized clinical trial of a postdischarge pharmaceutical care program vs regular follow-up patients with heart failure. Farm Hosp. 2006;30(6):328-342.

23. Laramee A, Levinsky S, Sargent J, Ross R, Callas P. Case management in a heterogeneous congestive heart failure population: a randomized controlled trial. Arch Intern Med. 2003;163(7):809-817.

24. Rich M, Gray D, Beckham V, Wittenberg C, Luther P. Effect of a multidisciplinary intervention on medication compliance in elderly patients with congestive heart failure. Am J Med. 1996;101(3):270-276.

25. Jimmy B, Jimmy J. Patient medication adherence: measures in daily practice. Oman Med J. 2011;26(3):155-159.

26. Dunlay SM, Eveleth JM, Roger VL. Medications adherence among community-dwelling patients with heart failure. Mayo Clin Proc. 2011; 86(4):273-281.

27. Butler J, Arbogast PG, Daughterty J, Jain MK, Ray WA, Griffin MR. Outpatient utilization of angiotensin converting enzyme inhibitors among heart failure patients after hospital discharge. J Am Coll Cardiol. 2004;43(11):2036-2043.

28. Johnson G, Shalansky S. Predictors of refill non-adherence in patients with heart failure. Br J Clin Pharmcol. 2007;63(4):488-493.

29. Bouvy ML, Heerdink ER, Urquhart J, Grobbee DE, Hoes AW, Leufkens HG. Effect of a pharmacist led intervention on diuretic compliance in heart failure patients: a randomized controlled study. J Cardiol Fail. 2003;9(5):404-411.

30. Artinian NT, Harden JK, Kronenberg MW, et al. Pilot study of a web based compliance monitoring device for patients with congestive heart failure. Heart Lung. 2003;32(4):226-233.

31. Fulmer TT, Feldman PH, Kim TS, et al. An intervention study to enhance medication compliance in community-dwelling elderly individuals. J Gerontol Nurs. 1999;25(8):6-14.

32. Wu J, Corley D, Lennie T, Moser D. Effect of a medication-taking behavior feedback theory - based intervention on outcomes in patients with heart failure. J Card Fail. 2012;18(1):1-9.

33. Murray MD, Young J, Hoke S, et al. Pharmacist intervention to improve medication adherence in heart failure: a randomized trial. Ann Intern Med. 2007;146(10):714-725.

34. Lehane E, McCarthy G. Intentional and unintentional medication non-adherence: a comprehensive framework for clinical research and practice? A discussion paper. Int J Nurs Stud. 2007;44(8):1468-1477.

35. World Health Organization. Adherence to Long Term Therapies: Evidence for Action. Switzerland: World Health Organization; 2003. 
36. Wu JR, Moser DK, Lennie TA, Peden AR, Chen YC, Heo S. Factors influencing medication adherence in patients with heart failure. Heart Lung. 2008;37(1):8-16.

37. Cole JA, Norman H, Weatherby LB, Walker AM. Drug copayment and adherence in chronic heart failure: effect on cost and outcomes. Pharmacotherapy. 2006;26(8):1157-1164.

38. Cholowski K, Cantwell R. Predictors of medication compliance among older heart failure patients. Int J Older People Nur. 2007;2(4):250-262.

39. Bagchi AD, Esposito D, Kim M, Verdier J, Bencio D. Utilization of, and adherence to, drug therapy among medicaid beneficiaries with congestive heart failure. Clin Ther. 2007;29(8):1771-1783.

40. Granger BB, Ekman I, Granger CB, et al. Adherence to medication according to sex and age in the CHARM programme. Eur J Heart Fail. 2009;11(11):1092-1098.
41. Riegel B, Lee CS, Ratcliffe SJ, et al. Predictors of objectively measured medication nonadherence in adults with heart failure. Circ Heart Fail. 2012;5(4):430-436.

42. Riegel B, Carlson B. Facilitators and barriers to heart failure self-care. Patient Educ Couns. 2002;46(4):287-295.

43. Aggarwal B, Pender A, Mosca L, Mochari-Greenberger H. Factors associated with medication adherence among heart failure patients and their caregivers. J Nurs Edu Pract. 2015;5(3):22-27.

44. Hughes MC. Improving medication adherence. Nurs Incred Easy. 2012; $10(6): 56$.

45. American Telemedicine Association. What is Telemedicine? Washington, DC: American Telemedicine Association; 2012.

\section{Publish your work in this journal}

Research Reports in Clinical Cardiology is an international, peerreviewed, open access journal publishing original research, reports, editorials, reviews and commentaries on all areas of cardiology in the clinic and laboratory. The manuscript management system is completely online and includes a very quick and fair peer-review system.
Visit http://www.dovepress.com/testimonials.php to read real quotes from published authors. 